\title{
Investigation of the antibacterial activity of pioglitazone
}

This article was published in the following Dove Press journal:

Drug Design, Development and Therapy

26 September 2011

Number of times this article has been viewed

\author{
Majed M Masadeh' \\ Nizar M Mhaidat ${ }^{2}$ \\ Sayer I Al-Azzam² \\ Karem HAlzoubi ${ }^{2}$ \\ 'Department of Pharmaceutical \\ Technology; ${ }^{2}$ Department of Clinical \\ Pharmacy, Faculty of Pharmacy, Jordan \\ University of Science and Technology, \\ Irbid, Jordan
}

Purpose: To evaluate the antibacterial potential of pioglitazone, a member of the thiazolidinediones class of drugs, against Gram-positive (Streptococcus pneumoniae) and Gram-negative (Escherichia coli and Klebsiella pneumoniae) bacteria.

Methods: Susceptibility testing was done using the antibiotic disk diffusion method and the minimal inhibitory concentration (MIC) of pioglitazone was measured according to the broth micro incubation standard method.

Results: Pioglitazone induced a dose-dependent antibacterial activity in which the optimal concentration was $80 \mu \mathrm{M}$. Furthermore, results indicated that while E. coli was sensitive $(\mathrm{MIC}=31.25 \pm 3.87 \mathrm{mg} / \mathrm{L})$ to pioglitazone-induced cytotoxicity, S. pneumoniae and K. pneumoniae were resistant $(\mathrm{MIC}=62.5 \pm 3.77 \mathrm{mg} / \mathrm{L}$ and $\mathrm{MIC}=62.5 \pm 4.14 \mathrm{mg} / \mathrm{L}$, respectively). Moreover, pretreatment of bacteria with a suboptimal concentration of pioglitazone $(40 \mu \mathrm{M})$ before adding amoxicillin, cephalexin, co-trimoxazole, or ciprofloxacin enhanced the antibacterial activity of all agents except co-trimoxazole. This enhancing effect was particularly seen against $K$. pneumoniae.

Conclusion: These results indicate the possibility of a new and potentially important pioglitazone effect and the authors' ongoing studies aim to illustrate the mechanism(s) by which this antibacterial effect is induced.

Keywords: pioglitazone, susceptibility testing, antibiotics, diabetes

\section{Introduction}

Thiazolidinediones (TZDs), also known as glitazones, are a relatively new class of oral hypoglycemic, insulin-sensitizing drugs that are used clinically as third-line agents in the management of diabetes mellitus type 2 as they help restore peripheral insulin sensitivity. ${ }^{1}$ TZDs activate peroxisome proliferator-activated receptor gamma (PPAR- $\gamma$ ), a nuclear receptor that regulates the production of proteins involved in glucose and lipid homeostasis, and, to a lesser extent, PPAR $-\gamma \cdot{ }^{1-2}$

Pioglitazone is one member of the thiazolidinedione family. It is an oral hypoglycemic agent dosed at $15-30 \mathrm{mg} /$ day. The maximum recommended dose for pioglitazone is $45 \mathrm{mg} /$ day, which is associated with peak plasma drug concentrations of pioglitazone in the range of $1050-1329 \mu \mathrm{g} / \mathrm{L} \cdot{ }^{3-5}$ In addition to its hypoglycemic effect, pioglitazone has many other effects. ${ }^{6-8}$ For example, like other TZDs, pioglitazone has been shown to reduce plasma levels of several inflammatory mediators and tissue-remodeling enzymes. The anti-inflammatory effects of TZDs are also evident in patients with classical metabolic syndrome since they reduce the white blood cell count, P-selectin-positive platelets, acute-phase inflammatory proteins, C-reactive protein, serum amyloid A, and fibrinogen.
Correspondence: Majed M Masadeh Faculty of Pharmacy, Jordan University of Science and Technology, Irbid 22 1 I 0 , Jordan

Tel +962 27201000

Fax +962 27201075

Email mmmasadeh@just.edu.jo 
Thus, TZDs have shown efficacy in inflammatory diseases such as psoriasis, ulcerative colitis, and nonalcoholic steatohepatitis. ${ }^{9}$

Previous attempts have been made to investigate the potential antibacterial effect of TZDs and it has been shown that some novel thiazolidine-2,4-dione derivatives possess antibacterial and antifungal activities. ${ }^{10,11}$ However, no study has tested whether the widely used antidiabetic TZDs have antibacterial activity. Therefore, in this study, the authors evaluated the possibility that pioglitazone, the most widely used TZD, possesses antibacterial activity against important Gram-positive microorganisms such as Streptococcus pneumoniae, and Gram-negative microorganisms such as Escherichia coli and Klebsiella pneumoniae.

\section{Material and methods}

\section{Microbial culture and growth conditions}

The antibacterial activities of pioglitazone and pioglitazone at a suboptimal concentration of $40 \mu \mathrm{M}$ in combination with other agents (amoxicillin, cephalexin, co-trimoxazole, and ciprofloxacin) were compared against the reference bacteria, which included E. coli ATCC 25922 and clinical isolates $S$. pneumoniae and $K$. pneumoniae obtained from the microbiology laboratory at King Abdullah University Hospital, Ar Ramtha, Jordan.

The organisms were stored at $-70^{\circ} \mathrm{C}$ in $\mathrm{BBL}^{\mathrm{TM}}$ Trypticase $^{\mathrm{TM}}$ Soy Broth with 20\% glycerol (Becton Dickinson and Company, Cockeysville, MD). Once ready for batch susceptibility testing, organisms were thawed and passed three times to assure purity and viability. Using the agar plate dilution method, the minimum inhibitory concentrations (MICs) were determined in accordance with the Clinical and Laboratory Standards Institute (CLSI). ${ }^{12}$

Antibiotic solutions were prepared on the day of use according to manufacturer recommendations. Concentrations that ranged from 31.25 to $500 \mu \mathrm{g} / \mathrm{mL}$ were tested. Serial dilutions of two folds were added to molten $\mathrm{BBL}^{\mathrm{TM}}$ Muller Hinton Gold II agar (Becton Dickinson and Company). After that, the agar plates were slightly cooled and dried. Thereafter, a steer replicator was used to place aliquots containing approximately $5 \times 10^{4}$ colony-forming units per drop for each of the three test strains of E. coli, S. pneumoniae, and $K$. pneumoniae. Plates were read in duplicate after incubation for 18 hours at $35^{\circ} \mathrm{C}$. MIC was defined as the lowest concentration at which there was no growth, or a faint haze or fewer than three discrete colonies. The breakpoints indicated in the last edition of tables of the National Committee for CLSI ${ }^{13}$ were used to determine susceptibility and resistance.
Table I Dose titration of pioglitazone against Escherichia coli, Streptococcus pneumoniae and Klebsiella pneumoniae

\begin{tabular}{llll}
\hline Pioglitazone & \multicolumn{3}{l}{ Diameter zone of inhibition $(\mathbf{m m})$} \\
\cline { 2 - 4 } conc $(\mu \mathrm{M})$ & E. coli & S. pneumoniae & K. pneumoniae \\
\hline 5 & 0 & 0 & 0 \\
10 & 0 & 0 & 0 \\
20 & 0 & 0 & 0 \\
40 & 0 & 0 & 0 \\
50 & 17 & 0 & 0 \\
60 & 18 & 10 & 9 \\
70 & 19 & 12 & 17 \\
80 & 26 & 20 & 19 \\
100 & 21 & 19 & 18 \\
150 & 19 & 14 & 12 \\
200 & 19 & 14 & 12 \\
\hline
\end{tabular}

Abbreviation: conc, concentration.

\section{Antimicrobial susceptibility test}

Sterile $5 \mathrm{~mm}$ diameter filter paper discs that were impregnated with different concentrations of amoxicillin, cephalexin, co-trimoxazole, and ciprofloxacin, were placed in duplicate on Mueller Hinton agar. In some experiments, pioglitazone at a suboptimal concentration of $40 \mu \mathrm{M}$ was added to the media. The surface was then spread with $0.2 \mathrm{~mL}$ of microorganism culture (about $10^{8}$ cells $/ \mathrm{mL}$ ) and the plates were incubated for 24 hours at $37^{\circ} \mathrm{C}$. The experiments were carried out in duplicate. The results (mean of three independent experiments) were recorded by measuring the zones of growth inhibition surrounding the discs.

\section{Determination of MIC}

The MIC was determined using serial dilution method according to the procedures of the National Committee for Clinical Laboratory Standards. ${ }^{13}$ Briefly, stock solutions of amoxicillin, cephalexin, co-trimoxazole, and ciprofloxacin were sterilized by passing them through a pyrogenic filter. Solutions were then serially diluted to a range of concentrations. Each well of a 96-well plate was prepared by dispensing $100 \mu \mathrm{L}$ of an appropriate medium, an antibiotic (amoxicillin, cephalexin, co-trimoxazole, or ciprofloxacin), and $20 \mu \mathrm{L}$ of the inoculum. A standard nutrient broth (Muller Hinton [Sigma-Aldrich, MI, USA]) was used. Turbidity was the sign that indicated the growth of microorganisms, whereas clear wells indicated absence of bacterial growth.

Table 2 Minimal inhibitory concentration (MIC) of pioglitazone

\begin{tabular}{lll}
\hline MIC $(\mathrm{mg} / \mathrm{L})$ & \\
\hline E. coli & S. pneumoniae & K. pneumoniae \\
\hline $31.25( \pm 3.87 \mathrm{mg} / \mathrm{L})$ & $62.5( \pm 3.77 \mathrm{mg} / \mathrm{L})$ & $62.5( \pm 4.14 \mathrm{mg} / \mathrm{L})$ \\
\hline
\end{tabular}

Abbreviations: E. coli, Escherichia coli; S. pneumoniae, Streptococcus pneumoniae; K. pneumoniae, Klebsiella pneumoniae. 
Table 3 Cytotoxicity of pioglitazone and other clinically used antibiotics

\begin{tabular}{llllll}
\hline Bacteria species & Pioglitazone & Amoxicillin & Cephalexin & Co-trimoxazole & Ciprofloxacin \\
\hline E. coli & 0 & 9 & 19 & 22 & 31 \\
K. pneumoniae & 0 & 0 & 0 & 0 & 32 \\
S. pneumoniae & 0 & 8 & 24 & 25 & 35 \\
\hline
\end{tabular}

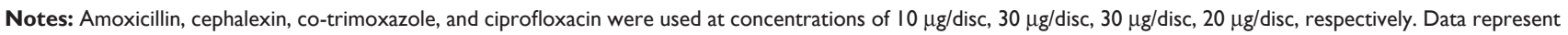
diameters of zone of inhibition (in $\mathrm{mm}$ ).

Abbreviations: E. coli, Escherichia coli; S. pneumoniae, Streptococcus pneumoniae; K. pneumoniae, Klebsiella pneumoniae.

For each experiment, a sterility check ( $50 \%$ dimethyl sulfoxide [DMSO; Sigma-Aldrich, MI, USA] and medium), negative control (50\% DMSO, medium and inoculum), and positive control (50\% DMSO, medium, inoculum, and ciprofloxacin) were included. The microtiter plates were incubated at $37^{\circ} \mathrm{C}$ for 24 hours and were examined for growth in daylight. The MIC of the preparations was the lowest concentration in the medium that completely inhibited the visible growth. The solvent value was deducted accordingly to obtain the final results of activity. All experiments were performed in triplicate.

\section{Chemicals}

Amoxicillin, cephalexin, co-trimoxazole, and ciprofloxacin were a generous gift from Al-HIKMA pharmaceuticals (Amman, Jordan). Pioglitazone was a generous gift from Dar Al-Dawa pharmaceuticals (Amman, Jordan). All drugs were used as pure material.

\section{Results}

Results (shown in Table 1) revealed that pioglitazone induced a dose-dependent antibacterial activity against the reference bacteria. An inhibition zone of $10 \mathrm{~mm}$ was chosen as representative of bacterial susceptibility to the compound. The optimal concentration of pioglitazone was $80 \mu \mathrm{M}$. As shown in Table 2, E. coli, S. pneumoniae, and $K$. pneumoniae had MIC values to pioglitazone of $31.25 \pm 3.87 \mathrm{mg} / \mathrm{L}, 62.5 \pm 3.77 \mathrm{mg} / \mathrm{L}$, and $62.5 \pm 4.14 \mathrm{mg} / \mathrm{L}$, respectively. These MIC values, although indicating the antibacterial activity of pioglitazone, are considered relatively high compared with those of standard antibiotics. Thus, E. coli, S. pneumoniae, and K. pneumoniae are considered resistant to pioglitazone alone.

The possibility that pioglitazone might enhance the activity of other clinically used antibacterial agents was then considered. Pioglitazone at suboptimal concentra- tion of $40 \mu \mathrm{M}$ and other agents (amoxicillin, cephalexin, co-trimoxazole, and ciprofloxacin) were used to assess the antibacterial activity against the reference bacteria. As shown in Table 3, pioglitazone alone, at suboptimal concentration, did not induce antibacterial effect against any of the bacteria. While all bacteria were resistant to amoxicillin, they were sensitive to ciprofloxacin-induced cytotoxicity. E. coli and $S$. pneumoniae were moderately sensitive to co-trimoxazole and cephalexin. Furthermore, pretreatment of bacteria with suboptimal concentration of pioglitazone $(40 \mu \mathrm{M})$ enhanced the antibacterial activity of all agents except co-trimoxazole. This enhancing effect of pioglitazone was particularly seen against $K$. pneumoniae (Tables 4 and 5).

\section{Discussion}

The emergence of drug resistance along with the poor compliance of patients, undesirable adverse drug effects, and the significant elevation of cost due to combination therapy, exposes the strong demand for new therapeutic regimens having the same or higher beneficial properties of antibiotics but with reduced adverse effects. Pioglitazone, a member of the thiazolidinediones class of medications, has been shown to induce different effects such as hypoglycemic effect in diabetic patients,,${ }^{14}$ anti-inflammatory effects, ${ }^{7,8}$ and to improve vascular endothelial function and microalbuminuria. ${ }^{14}$ In this study, the authors have shown, for the first time, a dose-dependent antibacterial activity of pioglitazone against reference bacteria. For clinical isolates, only E. coli was sensitive to pioglitazone actions, while both Gram-positive $S$. pneumoniae and Gram-negative $K$. pneumoniae were resistant. Furthermore, pretreatment of bacterial cultures with pioglitazone enhanced the antibacterial activity of amoxicillin, cephalexin, and ciprofloxacin but not that of co-trimoxazole.

Table 4 Effect of pioglitazone $(P)$ on other clinically used antibiotics - induced cytotoxicity

\begin{tabular}{|c|c|c|c|c|}
\hline \multicolumn{5}{|c|}{ Diameter zone of inhibition $(\mathrm{mm})$} \\
\hline \multicolumn{5}{|c|}{ Dlameter zone or innidicion (mm) } \\
\hline E. coli & 15 & 19 & 22 & 37 \\
\hline K. pneumoniae & 10 & 8 & 7 & 42 \\
\hline S. pneumoniae & 12 & 28 & 30 & 39 \\
\hline
\end{tabular}


Table 5 Minimal inhibitory concentration (MIC) of pioglitazone $(P)$ combined with other antibiotics

\begin{tabular}{|c|c|c|c|c|c|c|c|c|}
\hline \multirow[t]{2}{*}{ Bacteria species } & \multicolumn{8}{|c|}{ MIC (mg/L) } \\
\hline & Amox & Amox $+\mathbf{P}$ & Cepha & Cepha + P & Co-trimoxazole & Co-trimoxazole $+\mathrm{P}$ & Cipro & Cipro + P \\
\hline E. coli & 250 & 125 & 250 & 125 & 125 & 125 & 250 & 125 \\
\hline K. pneumoniae & 500 & 250 & 500 & 250 & 125 & 62.5 & 250 & 125 \\
\hline S. pneumoniae & 500 & 250 & 125 & 31.25 & 125 & 62.5 & 125 & 62.5 \\
\hline
\end{tabular}

Abbreviations: E. coli, Escherichia coli; S. pneumoniae, Streptococcus pneumoniae; K. pneumoniae, Klebsiella pneumoniae; amox, amoxicillin; cepha, cephalexin; cipro, ciprofloxacin.

Previous studies have shown that thiazolidinediones induce their hypoglycemic effect, anti-inflammatory effects, and improved vascular endothelial function via transcription through their direct activation of the nuclear receptor PPAR- $\gamma,{ }^{9,15}$ Since this receptor lacks prokaryotes, it is highly unlikely that the antibacterial activity of pioglitazone can be attributed to a known mechanism of action of thiazolidinediones. However, pioglitazone has been shown to promote cytotoxicity, suppress growth, and induce apoptosis through enhancing cell death via the tumor necrosis factorrelated mechanism. ${ }^{16}$ It has also been shown to induce a rapid production of reactive oxygen species (ROS) unrelated to the PPAR- $\gamma .{ }^{17,18}$ This production of ROS was associated with the cytotoxic effect of pioglitazone. ${ }^{17,18}$ It is possible that the currently reported enhanced antibacterial activity of common antibiotics is related to the generation of ROS induced by pioglitazone. However, further work is needed to characterize such possible mechanisms.

In concordance with the authors' results, novel thiazolidine-2, 4-dione derivatives have been synthesized and their antimicrobial activity has been examined in a number of studies. In one study, phenylethylsulfanyl-1,3-thiazolothiazolidine-2,4-dione derivatives (VII a-f, VIII a-f) and 5-methyl-[1,2,4]triazolyl-sulfanyl-1,3-thiazolo-thiazolidine-2,4-dione derivatives (IX a-f, $\mathrm{X}$ a-f) were synthesized and investigated for their antibacterial and antifungal activities against $S$. aureus, methicillin-resistant Staphylococcus aureus, Bacillus subtilis, E. coli, and Candida albicans. The derived compounds were found to be active against these bacteria. ${ }^{10}$ In another study, the antimicrobial activity of these derivatives was evaluated against B. subtilis (Grampositive), Pseudomonas aeruginosa (Gram-negative), and Streptomyces spp. where they displayed different degrees of antimicrobial activities or inhibitory actions. ${ }^{11}$ In the present study, it was shown that pioglitazone possesses a dose-dependent antibacterial activity against Grampositive (S. pneumoniae) and Gram-negative (E. coli and K. pneumoniae) bacteria. This antibacterial activity of pioglitazone was manifested as enhanced antibacterial activity of standard antibiotics including amoxicillin, cephalexin, and ciprofloxacin against $S$. pneumoniae, E. coli, and $K$. pneumoniae.

These results raise the possibility of a new and important potential effect of pioglitazone. The authors' ongoing studies aim to illustrate the mechanism(s) by which pioglitazone induces its antibacterial effect.

\section{Acknowledgment}

The authors would like to acknowledge Jordan University of Science and Technology, Irbid, Jordan, for financial support (grant numbers 73-2006 and 226-2009).

\section{Disclosure}

The authors declare no conflicts of interest in relation to this work.

\section{References}

1. Gillies PS, Dunn CJ. Pioglitazone. Drugs. 2000;60(2):333-343; discussion 344-345.

2. Smith U. Pioglitazone: mechanism of action. Int J Clin Pract Suppl. 2001;121:13-18.

3. Wittayalertpanya S, Chompootaweep S, Thaworn N. The pharmacokinetics of pioglitazone in Thai healthy subjects. JMed Assoc Thai. 2006; 89(12):2116-2122

4. Budde K, Neumayer HH, Fritsche L, Sulowicz W, Stompôr T, Eckland D. The pharmacokinetics of pioglitazone in patients with impaired renal function. Br J Clin Pharmacol. 2003;55(4):368-374.

5. Christensen ML, Meibohm B, Capparelli EV, Velasquez-Mieyer P, Burghen GA, Tamborlane WV. Single- and multiple-dose pharmacokinetics of pioglitazone in adolescents with type 2 diabetes. J Clin Pharmacol. 2005;45(10):1137-1144.

6. Wada K, Kamisaki Y. Anti-inflammatory effect of PPARgamma agonists: basics and clinical applications. Nihon Rinsho. 2010;68(2): 278-283. Japanese.

7. Araki A. Dementia and insulin resistance in patients with diabetes mellitus. Nihon Rinsho. 2010;68(3):569-574. Japanese.

8. Gervois P, Fruchart JC, Staels B. Inflammation, dyslipidaemia, diabetes and PPars: pharmacological interest of dual PPARalpha and PPARgamma agonists. Int J Clin Pract Suppl. 2004;143:22-29.

9. Buckingham RE. Thiazolidinediones: Pleiotropic drugs with potent antiinflammatory properties for tissue protection. Hepatol Res. 2005;33(2): $167-170$.

10. Menteşe A, Ceylan-Unlüsoy M, Bozdağ-Dündar O, Altanlar N, Ertan R. Synthesis and antimicrobial activity of some novel thiazolidine-2, 4-dione derivatives. Arzneimittelforschung. 2009;59(12):659-665.

11. El-Sayed WA, Abdel-Monem YK, Yousif NM, Tawfek N, Shaaban MT, Abdel-Rahman AA. Antimicrobial activity of new 2,4-disubstituted thiazolidinone derivatives. Z Naturforsch C. 2009;64(11-12): $785-789$ 
12. Clinical and Laboratory Standards Institute (CLSI). Performance standards for antimicrobial susceptibility testing. M100-S20. Villanova, PA: 2010. Available from: http://www.clsi.org/source/orders/free/m100s20.pdf" www.clsi.org/source/orders/free/m100-s20.pdf. Accessed 14 September 2011

13. CLSI. Methods for dilution antimicrobial susceptibility test for bacteria that grow aerobically. Approved standard. Eighth edition. Villanova, PA: 2009. Available from http://www.clsi.org/source/orders/free/m07-a8.pdf. Accessed 14 September 2011.

14. Kalaitzidis RG, Sarafidis PA, Bakris GL. Effects of thiazolidinediones beyond glycaemic control. Curr Pharm Des. 2009;15(5):529-536.

15. Houseknecht KL, Cole BM, Steele PJ. Peroxisome proliferator-activated receptor gamma (PPARgamma) and its ligands: a review. Domest Anim Endocrinol. 2002;22(1):1-23.
16. Göke R, Göke A, Göke B, El-Deiry WS, Chen Y. Pioglitazone inhibits growth of carcinoid cells and promotes TRAIL-induced apoptosis by induction of p21waf1/cip1. Digestion. 2001;64(2):75-80.

17. Pérez-Ortiz JM, Tranque P, Burgos M, Vaquero CF, Llopis J. Glitazones induce astroglioma cell death by releasing reactive oxygen species from mitochondria: modulation of cytotoxicity by nitric oxide. Mol Pharmacol. 2007;72(2):407-417.

18. Pérez-Ortiz JM, Tranque P, Vaquero CF, et al. Glitazones differentially regulate primary astrocyte and glioma cell survival. Involvement of reactive oxygen species and peroxisome proliferator-activated receptor-gamma. J Biol Chem. 2004;279(10):8976-8985.

\section{Publish your work in this journal}

Drug Design, Development and Therapy is an international, peerreviewed open-access journal that spans the spectrum of drug design and development through to clinical applications. Clinical outcomes, patient safety, and programs for the development and effective, safe, and sustained use of medicines are a feature of the journal, which has also been accepted for indexing on PubMed Central. The manuscript management system is completely online and includes a very quick and fair peer-review system, which is all easy to use. Visit http://www.dovepress.com/testimonials.php to read real quotes from published authors.

Submit your manuscript here: http://www.dovepress.com/drug-design-development-and-therapy-journal 